Supporting Information

High-loading nickel cobaltate nanoparticles anchored on

three-dimensional $\mathrm{N}$-doped graphene as an efficient bifunctional

\title{
catalyst for lithium-oxygen batteries
}

Hao Gong, Hairong Xue, Tao Wang, Hu Guo, Xiaoli Fan, Li Song, Wei Xia and

Jianping $\mathrm{He}^{*}$

College of Materials Science and Technology, Jiangsu Key Laboratory of Materials and Technology for Energy Conversion, Nanjing University of Aeronautics and Astronautics, Nanjing 210016, PR China.

*Corresponding authors:

Prof. Jianping He, Tel: +86 2552112906 Fax: +86 25 52112626, E-mail: jianph@nuaa.edu.cn. 

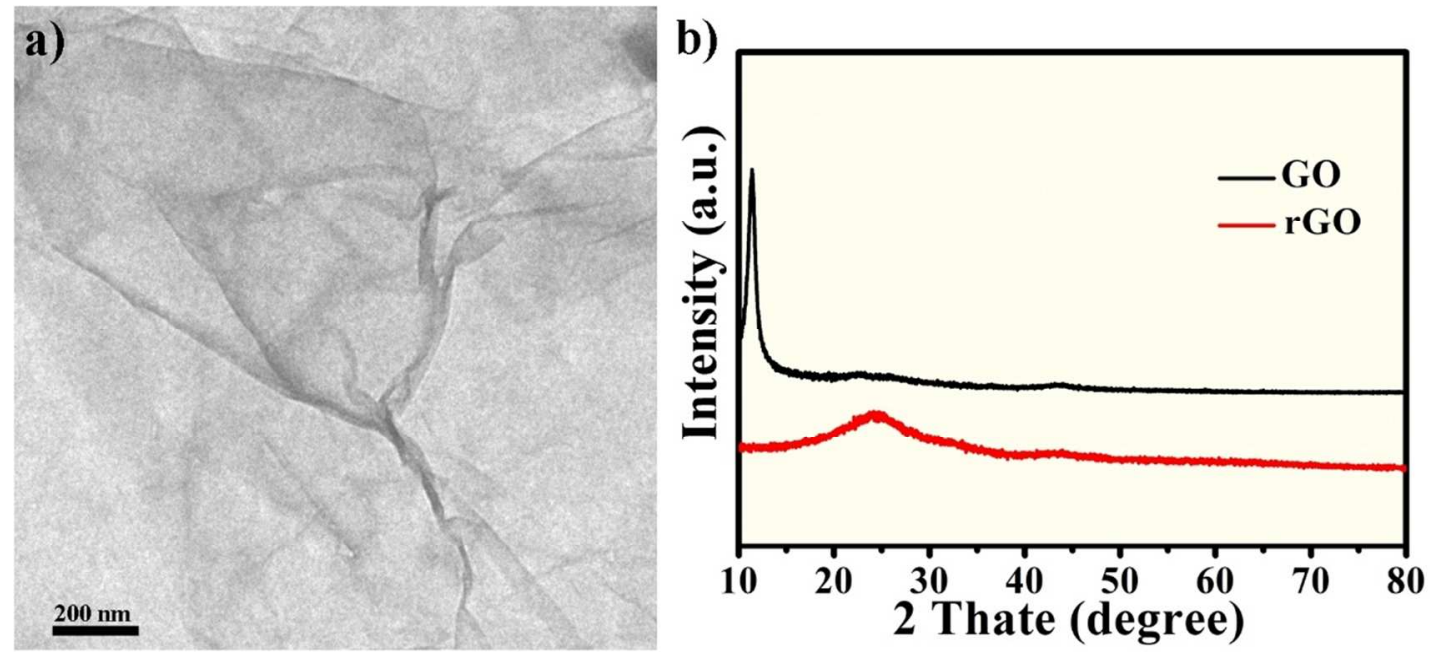

Figure S1. (a) the TEM image of the GO; (b) the XRD-pattern of the GO obtained through a modified Hummers method, and the rGO reduced by the hydrothermal methods.

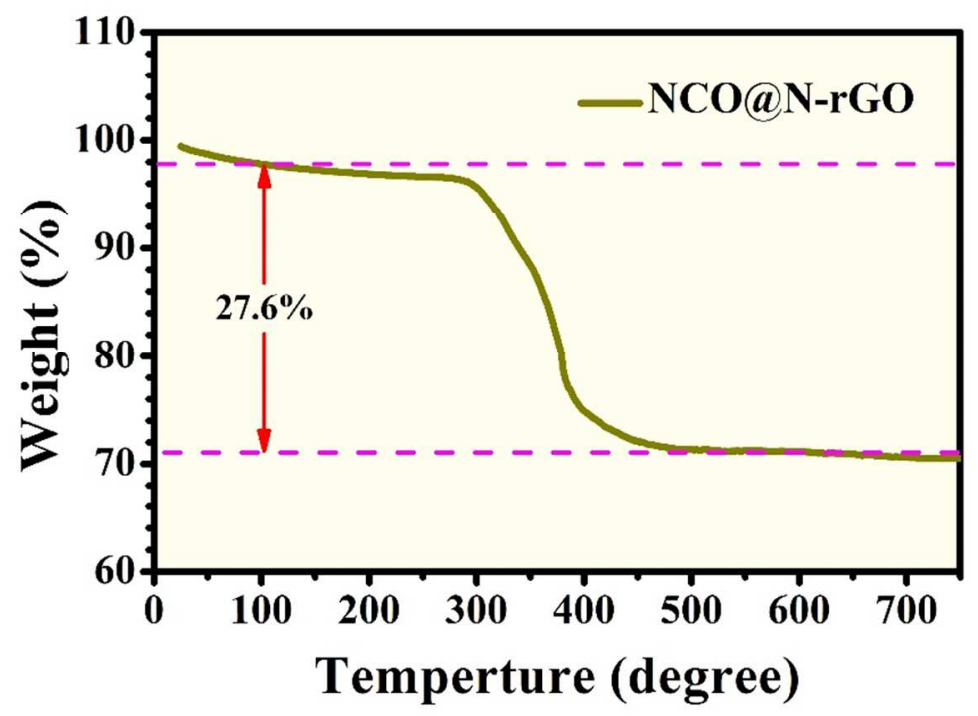

Figure S2. TG curves of NCO@N-rGO cathode subjected to a temperature range from room temperature to $900{ }^{\circ} \mathrm{C}$ under $\mathrm{O}_{2}$ atmosphere. 


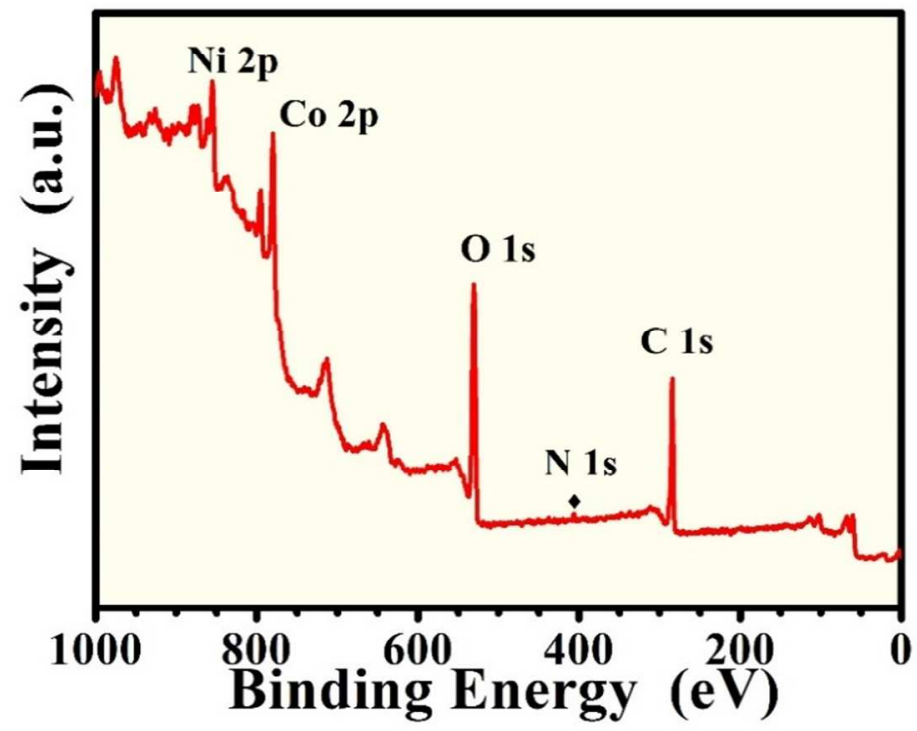

Figure S3. The XPS spectra of the NCO@N-rGO composites.

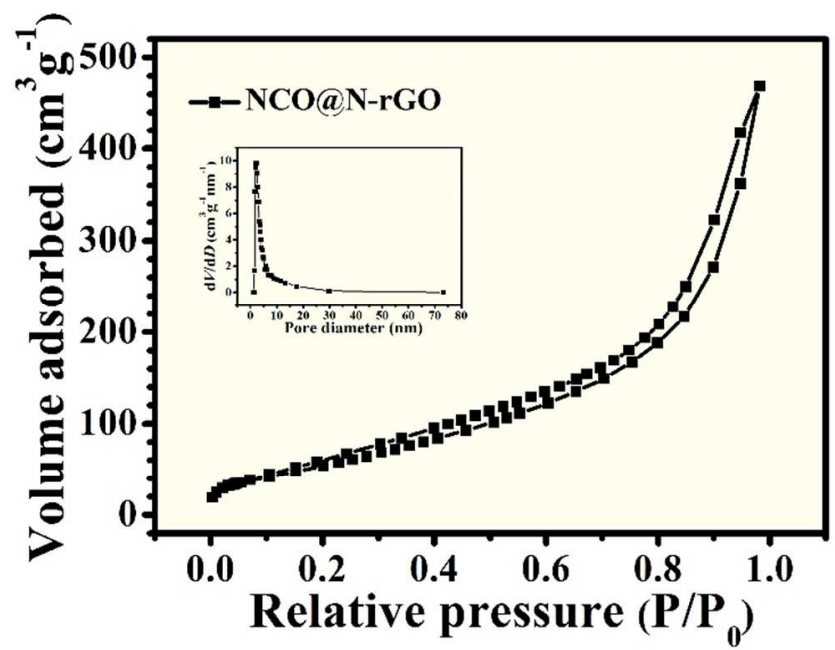

Figure S4. Nitrogen adsorption-desorption isotherms and pore-size distribution (inset) for NCO@N-rGO composites. 


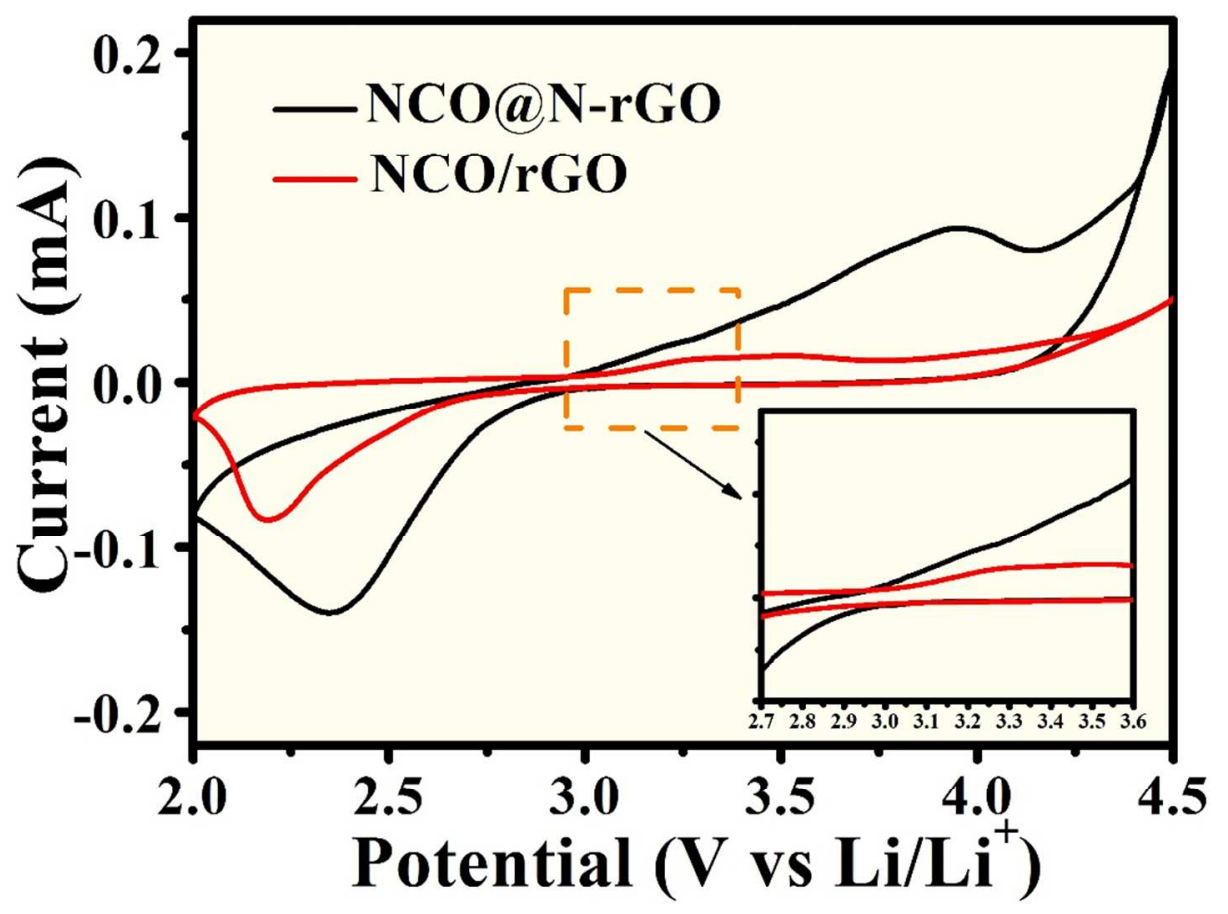

Figure S5. Cyclic voltammetry $(\mathrm{CV})$ curves of the $\mathrm{Li}^{-} \mathrm{O}_{2}$ cell with $\mathrm{NCO} / \mathrm{rGO}$ and $\mathrm{NCO} @ \mathrm{~N}-\mathrm{rGO}$ as a cathode in the electrolyte containing LiTFSI/TEGDME under $\mathrm{O}_{2}$ atmosphere at a scan rate of $0.1 \mathrm{mV} \mathrm{s}^{-1}$ and the inset is partial enlarged detail of the $\mathrm{CV}$.
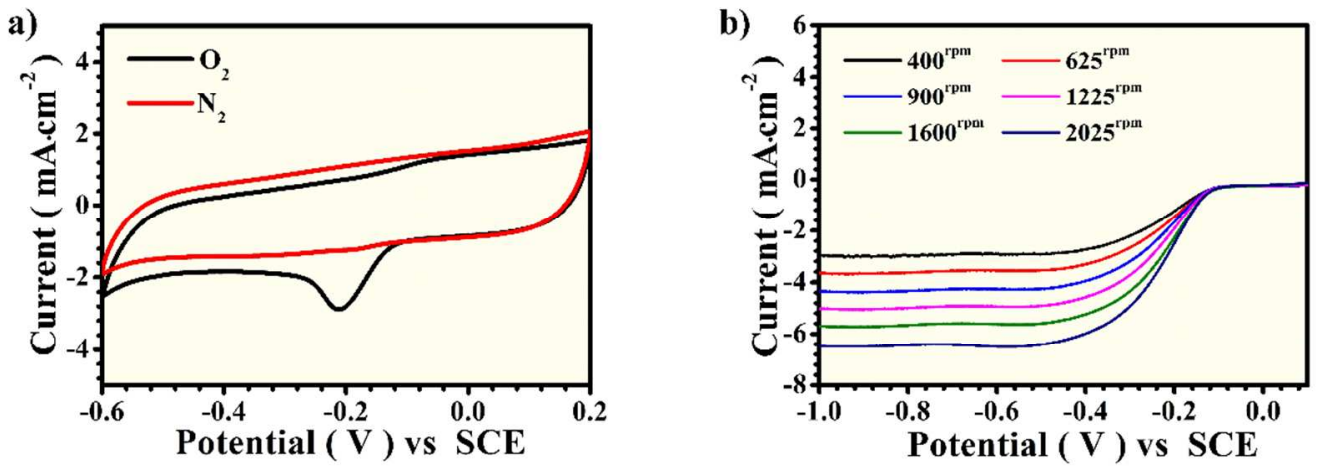

Figure S6. (a) Cyclic voltammetry (CV) of the NCO@N-rGO (b) RDE polarization curves at different rotating rates recorded for $\mathrm{NCO} @ \mathrm{~N}-\mathrm{rGO}$ composite in the $\mathrm{N}_{2}$ and $\mathrm{O}_{2}$-saturated 0.1 mol $\mathrm{L}^{-1} \mathrm{KOH}$. 